\title{
A Context-Based Analgesia Model in Rats: Involvement of Prefrontal Cortex
}

\author{
Lingchi $\mathrm{Xu}^{1} \cdot$ Yalan $\mathrm{Wan}^{2} \cdot$ Longyu Ma $^{1} \cdot \mathrm{Jie} \mathrm{Zheng}^{1} \cdot$ Bingxuan Han $^{1} \cdot$ \\ Feng-Yu Liu ${ }^{1} \cdot$ Ming Yi $^{1} \cdot$ You Wan ${ }^{1,3,4,5}$
}

Received: 21 February 2018/Accepted: 3 May 2018/Published online: 3 September 2018

(C) The Author(s) 2018

\begin{abstract}
Cognition and pain share common neural substrates and interact reciprocally: chronic pain compromises cognitive performance, whereas cognitive processes modulate pain perception. In the present study, we established a non-drug-dependent rat model of context-based analgesia, where two different contexts (dark and bright) were matched with a high $\left(52^{\circ} \mathrm{C}\right)$ or low $\left(48^{\circ} \mathrm{C}\right)$ temperature in the hot-plate test during training. Before and after training, we set the temperature to the high level in both contexts. Rats showed longer paw licking latencies in trials with the context originally matched to a low temperature than those to a high temperature, indicating successful establishment of a context-based analgesic effect in rats. This effect was blocked by intraperitoneal injection of naloxone (an opioid receptor antagonist) before the probe. The context-based analgesic effect also disappeared after optogenetic activation or inhibition of the bilateral infralimbic or prelimbic sub-region of the prefrontal cortex. In brief, we established a context-based, non-drug dependent, placebo-like
\end{abstract}

You Wan

ywan@hsc.pku.edu.cn

1 Neuroscience Research Institute, Peking University, Beijing 100083, China

2 Department of Clinical Medicine, Zhongshan School of Medicine, Sun Yat-Sen University, Guangzhou 510080, China

3 Department of Neurobiology, School of Basic Medical Sciences, Peking University, Beijing 100083, China

4 Key Laboratory for Neuroscience, Ministry of Education/ National Health and Family Planning Commission, Peking University, Beijing 100083, China

5 Co-innovation Center of Neuroregeneration, Nantong University, Nantong 226001, China analgesia model in the rat. This model provides a new and useful tool for investigating the cognitive modulation of pain.

Keywords Context-based analgesia - Placebo analgesia . Pain · Hot-plate test · Cognition modulation · Opioid system $\cdot$ Prefrontal cortex

\section{Introduction}

Pain and cognition inherently influence each other: pain can negatively affect cognitive performance, whereas cognitive modulation occurs in painful situations, for example as placebo and nocebo effects [1-3]. Recent studies have shown that cognitive functioning predicts the occurrence of post-surgical pain [4]. Some therapeutic interventions for pain, such as cognitive behavioral therapy, psychological consultation, and meditation, also target the cognitive-evaluative dimension of pain. However, the mechanisms underlying these interactions are not fully understood.

Efforts have been made to build rodent models of the cognitive modulation of pain, as well as cognition-mediated placebo analgesia [5-8], but these different training protocols are all based on analgesics that induce physiological changes and stress reactions. Based on the previous descriptions, in the present study we set out to build a rat model of context-based analgesia by matching two different contexts to a high or low testing temperature in the hotplate test. Then the involvement of the opioid system in the context-based analgesia was identified by naloxone injection.

Previous studies have shown that the activation of prefrontal glutamatergic neurons enhances recognition 
memory [9]. In addition, the activation of archaerhodopsin (Arch, a light-activated inhibitory proton pump) or channel rhodopsin 2 (ChR2, a light-activated excitatory cation channel) in parvalbumin (PV)-positive interneurons in the prefrontal cortex (PFC) decreases or increases pain responses, respectively [10]. Based on this, we hypothesized that the effects of cognition-mediated analgesia could be influenced by activation or inhibition of the PFC.

\section{Materials and Methods}

\section{Animals}

Adult female Sprague-Dawley rats weighing 250-300 g at the beginning of the experiment were provided by the Department of Experimental Animal Sciences, Peking University Health Science Center. Male rats were excluded because of the risk of the testicles being burned in the hotplate test. The rats were housed 4-6 per cage in a temperature- and light-controlled room under a 12:12 h light:dark cycle with water and food provided ad libitum. The animals were handled and habituated for 3-5 days before experiments. All experimental procedures were conducted in accordance with the guidelines of the International Association for the Study of Pain, complied with the ARRIVE guidelines [11], and were approved by the Animal Care and Use Committee of our University.

\section{Hot-Plate Test}

The hot-plate apparatus with a $30 \times 30 \times 30 \mathrm{~cm}^{3}$ Plexiglas chamber was located in a quiet room. The actual temperature of each test trial was as indicated below. The cut-off time was $30 \mathrm{~s}$ for the low temperature trials (LT, $48 \pm 0.5^{\circ} \mathrm{C}$ ), and $30 \mathrm{~s}$ for the high temperature trials (HT, $52 \pm 0.5^{\circ} \mathrm{C}$ ) to avoid possible plantar injury. The inter-trial interval was at least $10 \mathrm{~min}$.

\section{Establishment of a Rat Model of Context-Based Analgesia}

The training and testing procedures are shown in Fig. 1. The hot-plate test was performed in two different contexts in the same room: Context A was brightly lit (500-600 lumen) whereas Context B was dimly lit (1-2 lumen). After habituation to the apparatus for 2 days, baseline testing at the HT was performed on day 0. Paw licking latencies (PLLs) were averaged from 3 trials in each context. The trial sequence was pseudo-randomized so that no more than two consecutive trials were performed in the same context. The training phase was during days 1-7. Three groups (Test groups 1 and 2, and the Control group) were used to investigate the potential influences of different context and temperature combinations. These three groups were performed separately with three batches of animals. In Test group 1, Context A was matched with the LT and Context B with the HT. In Test group 2, Context A was matched with the HT and Context B with the LT. In the Control group, both contexts were matched with the HT. To strengthen the influence of contextual cues, the rat was placed in the corresponding context for $5 \mathrm{~min}$ before each trial as pre-exposure. On day 8 , a probe test was performed as on day 0 .

Training and testing (baseline and probes) were run by different experimenters to ensure testing blinded from groupings. This blinded approach also applied to all the subsequent experiments.

\section{Naloxone Injection in the Hot-Plate Test}

To determine whether opioids are involved in contextbased analgesia, manipulations on days $0-8$ were identical to those described above. Context A was matched with the LT and Context B with the HT. On day 9, animals were randomly divided into two groups. Half of the animals received an intraperitoneal (i.p.) injection of naloxone (an opioid antagonist) dissolved in normal saline (NS) at $5 \mathrm{mg} /$ $\mathrm{kg}$ body weight while the other half were injected with an equal volume of NS. Thirty minutes after injection, all rats received the probe test again as on day 8 . This was repeated on day 10 with each animal receiving an injection of the other solution (NS or naloxone). Data from days 9 and 10 were pooled as probe 2 for evaluating the effects of naloxone antagonism. The behavioral experimenters were blind to the solutions injected.

\section{Surgical Procedure}

Female Sprague-Dawley rats weighing 250-300 g were used in the optogenetic experiments. Each rat was anesthetized with $0.5 \%$ pentobarbital sodium $(100 \mathrm{mg} / \mathrm{kg}$, i.p.; Merck, Darmstadt, Germany) and placed in a stereotaxic frame. pAAV-CaMKIIa-hChR2-EYFP $\left(2.7 \times 10^{13}\right.$ viral genomes $(\mathrm{vg}) / \mathrm{mL})$ or pAAV-CaMKIIa-ArchT-EYFP $\left(2.7 \times 10^{13} \mathrm{vg} / \mathrm{mL}\right) \quad$ (Heyuan, Shanghai, China) was injected bilaterally into the prelimbic (PL; left side, AP +3.0, $\mathrm{ML}-1.8$, DV $-4.0 \mathrm{~mm}$, tilted $20^{\circ}$; right side, AP +3.0, ML -0.5 , DV $-3.5 \mathrm{~mm}$, vertical) or infralimbic cortex (IL; left side, AP +3.0, ML -1.8 , DV $-5.1 \mathrm{~mm}$, tilted $20^{\circ}$; right side, $\mathrm{AP}+3.0, \mathrm{ML}-0.5, \mathrm{DV}-4.6 \mathrm{~mm}$, vertical) through two small burr holes in the skull, where optical fibers $(\mathrm{NA}=0.37, \Phi=200 \mathrm{~mm}$; Fiblaser, Shanghai, China) were subsequently implanted. Expression of ChR2 and ArchT driven by the cell-type-specific promoter CaMKII was then observed in the glutamatergic PFC 


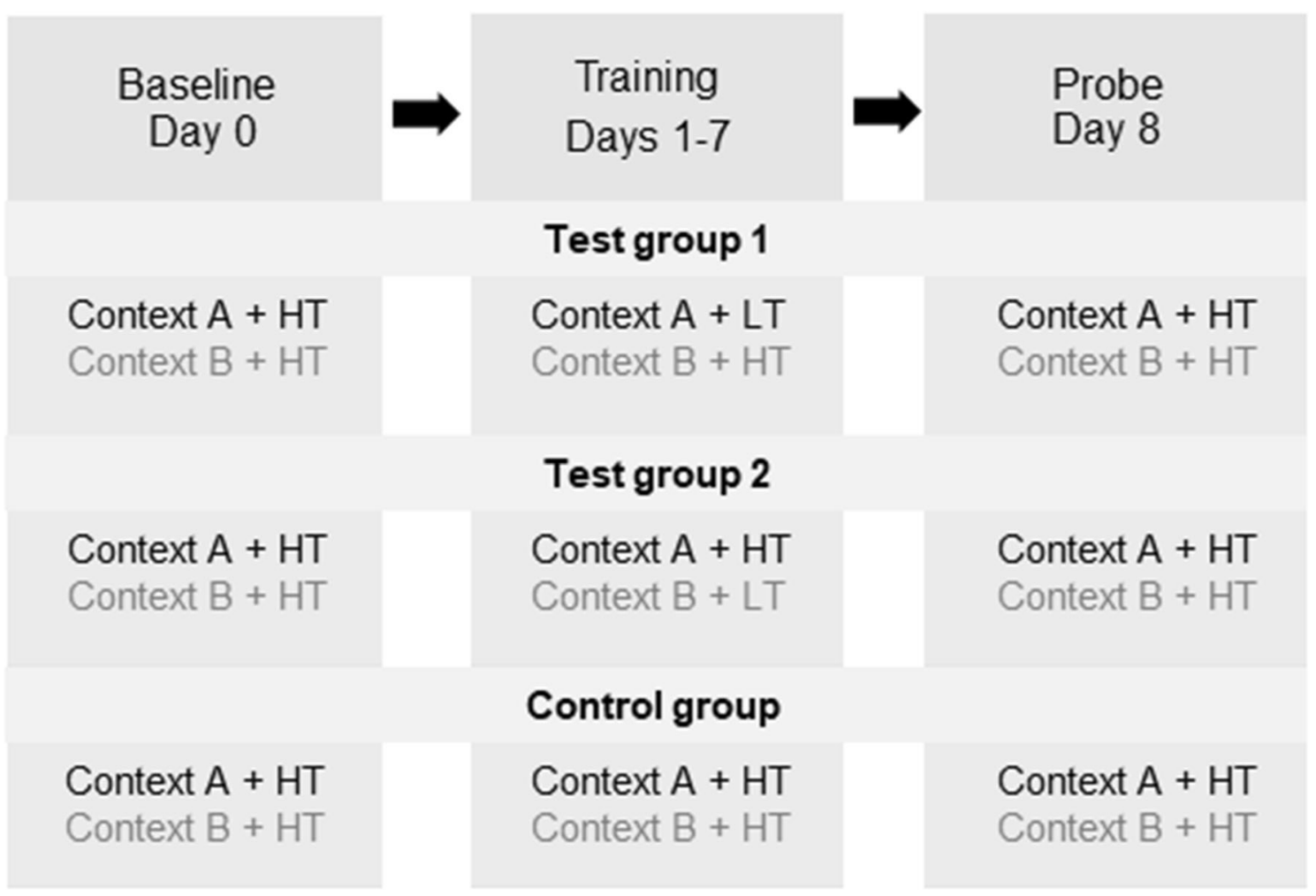

Fig. 1 Training and probe flowchart for the context-based analgesic effect in rats. Three groups were trained and tested in the three-phase experimental paradigm (Baseline: left column; Training: middle

neurons. The optical fibers were fixed to the skull with dental cement (New Century, Shanghai, China).

\section{Optogenetic Stimulation During the Hot-Plate Test}

The context-related behavioral training tests were performed 30 days later to allow for viral expression. The training paradigms were identical to those described above. Context A was matched with the LT and Context B with the HT. On day 9, animals were randomly divided into two groups of equal numbers. Light was delivered and the animal was put into the hot plate in one group, while the other group without light delivery in tests. A laser generator was connected to the bilateral optic fibers for at least $5 \mathrm{~min}$ before light delivery. The LED was turned off at the moment the rat licked its paw. This test was repeated on day 10 with exchange of the optogenetic stimulation in rats for self-contrast. hChR2- and ArchT-expressing neurons were stimulated with blue (25-ms pulses, $20 \mathrm{~Hz}, 6-9$ $\mathrm{mW}, 473 \mathrm{~nm}$ ) and yellow (25-ms pulses, $20 \mathrm{~Hz}, 6-9 \mathrm{~mW}$, $589 \mathrm{~nm}$ ) light during the hot-plate tests, respectively. The power output delivered was confirmed as 6-9 $\mathrm{mW}$ for each stimulation session using a power meter (Thorlabs, Newton, $\mathrm{NJ}$ ). column; Probe: right column). Contexts A (black) and B (grey) were in the same room but brightly- and dimly-lit, respectively. HT, high temperature $\left(52^{\circ} \mathrm{C}\right)$; LT, low temperature $\left(48^{\circ} \mathrm{C}\right)$.

\section{Brain Slice Preparation for Whole-Cell Patch- Clamp Recording}

Female Sprague-Dawley rats $(250-300 \mathrm{~g})$ were used for whole-cell patch clamp recording. The rats used for patch clamp were not the same as those used for the contextbased analgesia experiments $(n=5$ for the optogeneticactivation group; $n=6$ for the optogenetic-inhibition group). Each rat was anesthetized with pentobarbital sodium (40 mg/kg, i.p.). The brain was removed within 1 min and submerged in ice-cold artificial cerebrospinal fluid (aCSF) containing (in mmol/L): $125.0 \mathrm{NaCl}, 2.5 \mathrm{NaH}_{2} \mathrm{PO}_{4}$, $2.6 \mathrm{KCl}, 1.3 \mathrm{CaCl}_{2}, 21.0 \mathrm{NaHCO}_{3}, 0.9 \mathrm{MgCl}_{2}$, and 3.5 glucose. Coronal slices (thickness, $400 \mu \mathrm{m}$ ) that contained the PL/IL cortices were then cut on a Vibroslice $(1,000+$, Pelco 102; Ted Pella Inc., Redding, CA). The anatomical locations of the PL/IL cortices were confirmed on the basis of a rat brain atlas (Paxinos and Watson, 2007) and have been previously reported by our laboratory [12]. Before a single slice was transferred to a submerged recording chamber, slices were incubated in an oxygenated aCSF bath at room temperature for at least $1 \mathrm{~h}$. The chamber was perfused with aCSF (2-3 $\mathrm{mL} / \mathrm{min}$ ) using a pump (Peri-Star 291, World Precision Instruments, Sarasota, FL). All experiments were performed at room temperature [13]. 


\section{Whole-Cell Patch Clamp Recordings}

Each slice was viewed under a microscope (Axioskop Fsmot; Zeiss, Jena, Germany) equipped with infrared differential interference contrast optics. Pyramidal cells in the bilateral PL/IL cortices were identified through a $40 \times$ water-immersion lens.

Voltage and current signals were recorded from EYFPexpressing pyramidal cells using an Axon 200B amplifier (Axon Instruments, Union City, CA). Action potentials (APs) and inhibitory post-synaptic potentials (IPSPs) were recorded in the current-clamp mode. The holding voltage for the excitatory post-synaptic currents (EPSCs) and APs was the same $(-70 \mathrm{mV})$, and $0 \mathrm{mV}$ for recording IPSCs. The aCSF contained the following (in mmol/L): $124 \mathrm{NaCl}$, $26 \mathrm{NaHCO}_{3}, 3.0 \mathrm{KCl}, 1.0 \mathrm{NaH}_{2} \mathrm{PO}_{4}, 1.3 \mathrm{MgCl}_{2}, 1.5 \mathrm{CaCl}_{2}$, $20 \mathrm{D}$-glucose, saturated with $95 \% \mathrm{O}_{2}$ and $5 \% \mathrm{CO}_{2}$. The pipette solution contained (in $\mathrm{mmol} / \mathrm{L}$ ) 120 potassium gluconate, $10 \mathrm{KCl}, 4$ ATP-Mg, $0.3 \mathrm{GTP}, 10 \mathrm{HEPES}$, and 0.5 EGTA ( $\mathrm{pH} 7.2,270-280$ mOsm with sucrose).

\section{Histology}

The procedure was the same as in our previous report [14]. After all behavioral tests, each rat was deeply anesthetized and perfused with $4 \%$ paraformaldehyde in phosphate buffer. IL/PL sections ( $20 \mu \mathrm{m}$ thick) were cut coronally on a freezing microtome and used to identify the expression of EYFP and the locations of the optical fibers. Data from animals with incorrect location were excluded from further statistical analysis.

\section{Statistics}

All data are presented as the mean \pm SEM. Analysis of context-based analgesia and the effects of naloxone on the hot-plate test was performed with paired Student's $t$ test after a Gaussian distribution was found, except for the control group in Fig. 2, where the Wilcoxon matched pairs test was used. $P<0.05$ was considered to be statistically significant.

\section{Results}

\section{Establishment of Context-Based Analgesia Rat Model}

Baseline testing (day 0) at the HT revealed no differences in PLL between contexts in all three groups (Test group 1: $t=0.09, n=8$; Test group 2: $t=0.36, n=8$; Control group: $W=3$; all $P>0.05$ ) (Fig. 2).
During the 7 days of training, PLLs in the HT-matched context (Context B for Test group 1 and Context A for Test group 2) were shorter than those in the LT-matched context (Context A for Test group 1 and Context B for Test group 2, Fig. 2A, B). In the Control group in which the same temperature was used, PLLs were comparable between contexts (Fig. 2C).

In the probe trial on day 8 , the PLLs of rats that stayed in the context originally matched with the HT (Context B for Test group 1 and Context A for Test group 2) were significantly shorter than those with the LT (Context A for Test group 1 and Context B for Test group 2) (Test group 1: $t=4.61, P<0.01$; Test group 2: $t=2.94, P<0.05$ ) (Fig. 2A, B). In contrast, the PLLs in the control group did not significantly differ between contexts $(W=3, P>0.05)$ (Fig. 2C).

The PLLs of probe tests in the context matched with the LT (Context A for Test group 1 and Context B for Test group 2) were significantly longer than baseline (Test group 1: $t=2.65$; Test group 2: $t=2.94$, both $P<0.05$ ) (Fig. 2A, B), while the PLLs of probe tests in the context originally matched with the HT did not differ from baseline (Test group 1: $t=1.24$; Test group 2: $t=0.29$, both $P>0.05$ ) (Fig. 2A, B). These results indicate a clear cognition-mediated analgesic effect in the Test groups, induced by behavioral training with two contexts. In addition, different combinations of contexts and temperatures had no significant effect on the results.

\section{Naloxone Confirmation of Context-Based Analgesia}

It is well known that classical cognition-pain modulation is very similar to placebo analgesia, which is endogenously opioid-dependent $[6,15]$. So we next performed naloxone blockade experiments to determine whether the above context-based analgesia is dependent on cognitive modulation.

Behavioral training yielded a context-based analgesic effect similar to that described above (Fig. 3). Probe test on day 8 indicated a clear and stable difference in contextdependent pain perception between contexts $(t=2.58$, $P<0.05, n=10)$. The PLLs of probe test 1 in context A (matched with LT in training) were significantly longer than baseline $(t=3.06, P<0.05)$, which demonstrated a correlation between the context matched with the LT and context-based analgesia, while the PLLs in context B (matched with the HT in training) did not differ significantly between probe test 1 and baseline $(t=0.69$, $P>0.05)$.

Interestingly, injection of naloxone abolished this context-based analgesic effect $(n=10, \quad \mathrm{NS}: t=3.86$, $P<0.01$; naloxone: $t=0.90, P>0.05$ ) (Fig. 3B), suggesting that the opioid system is involved in mediating this 
Fig. 2 Establishment of the context-based analgesia model in rats. Three groups were trained and tested in the threephase experimental paradigm. Context A (black) was a bright room whereas Context B (grey) was a dim room. Paw licking latencies (PLLs) of Test group 1 (A), Test group 2 (B), and Control group $(\mathbf{C})$ in the baseline, training and probe phases are shown. The different contexts modulated pain perception with the same hot-plate temperature in the probe test. The context-based analgesic effect was indicated by the differential PLLs between baseline and the probe test with the same context and hot-plate temperature.

$n=8, * P<0.05, * * P<0.01$, paired $t$ test.
A

\section{Test group 1}
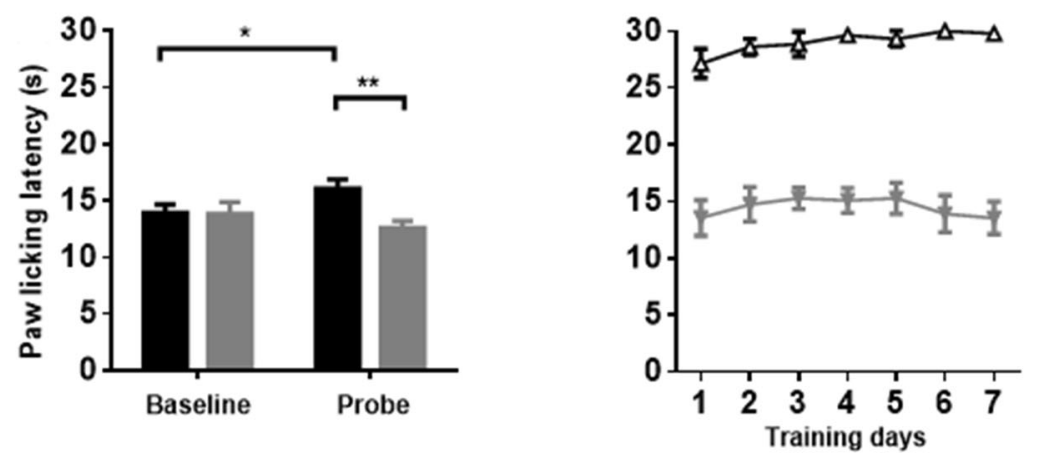

B

Test group 2
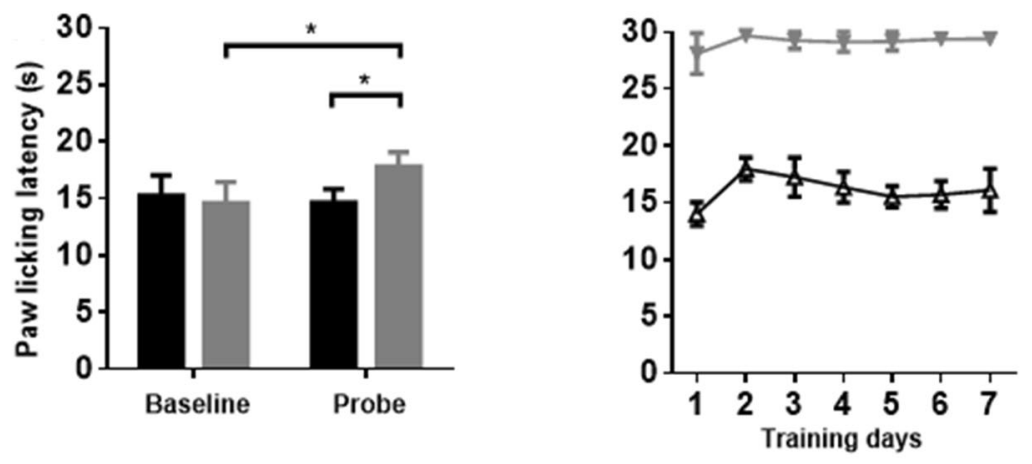

C

\section{Control group}

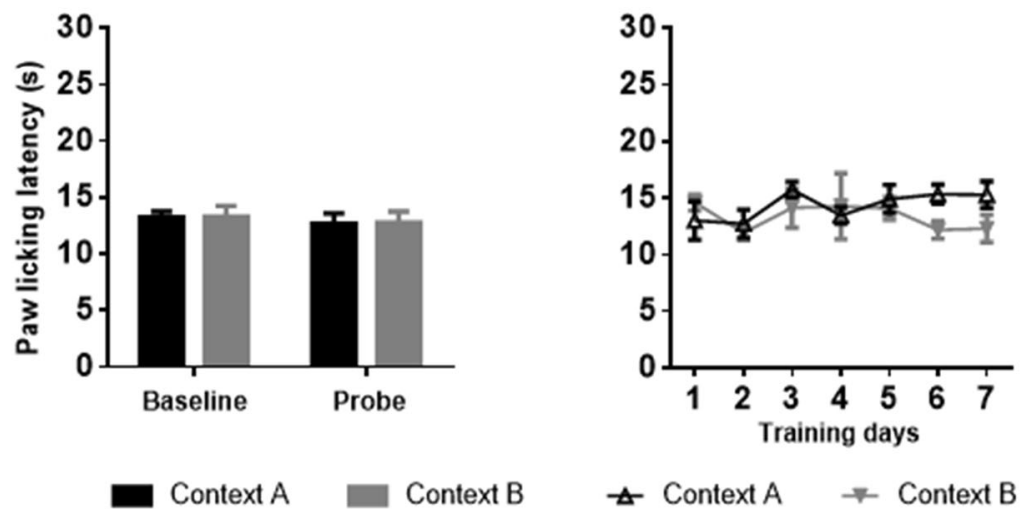

cognitive modulation of analgesia. Meanwhile, the PLLs (context A) in the saline group were longer than baseline $(t=2.50, P<0.05)$ (Fig. 3B). These results indicate that the context-induced analgesia effect depends on the endogenous opioid system.

\section{Effective Activation/Inhibition of Pyramidal Neu-} rons in PL/IL Cortices

Optogenetic manipulation with hChR2 and Arch has been widely used to activate or inhibit specific types of neurons. 


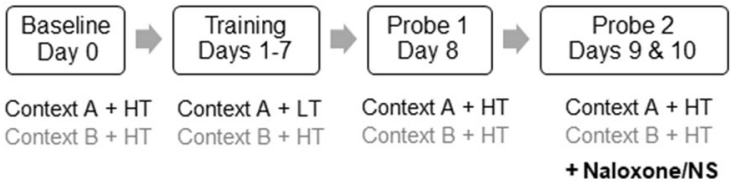

B

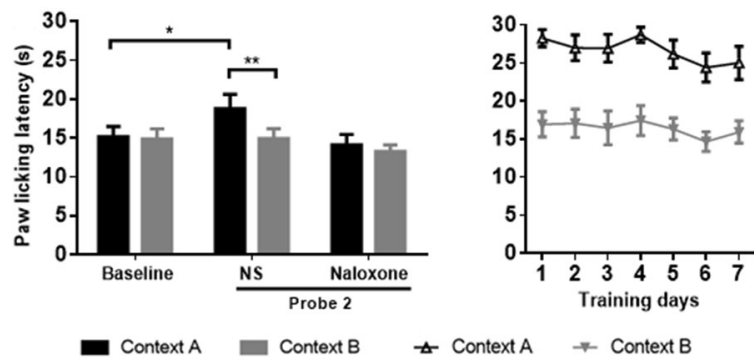

Fig. 3 Naloxone blockade of the context-based analgesic effect in rats. A Training and testing paradigm. B Naloxone injection affected PLLs in the hot-plate test. In contrast, the context-based analgesia was not influenced by saline injection. Context A, black; Context B, grey; HT, high temperature; LT, low temperature. $n=10,{ }^{*} P<0.05$, $* * P<0.01$, paired $t$ test.

The hChR2 or Arch gene can be selectively expressed in specific neurons with a neuronal type-specific promoter $[10,13,14,16]$. We also used fluorescent staining of pyramidal neurons to confirm the localization and expression of pAAV-CaMKIIa-hChR2-EYFP and pAAV-CaMKIIa-ArchT-EYFP in the bilateral PFC subregions PL and IL (Fig. 4B), as in our previous report [14].

In this study, whole-cell patch clamp recordings were performed to determine whether hChR2 and ArchT were expressed in glutamatergic neurons with the CaMKIIa promoter. The recordings from ArchT-expressing pyramidal neurons revealed that yellow-light $(589 \mathrm{~nm})$ stimulation not only evoked IPSCs and IPSPs, but also inhibited AP firing during current injection through the micropipette (Fig. 4C). hChR2-expressing glutamatergic neuronal activity was recorded in brain slices. Blue-light (473 nm) stimulation induced strictly pulse-locked APs in neurons (Fig. 4D). Thus, we confirmed the expression and function of hChR2 and ArchT in pyramidal neurons under the control of the CaMKIIa promotor.

\section{Optogenetic Activation of the PL or IL Cortex Eliminates the Context-Based Analgesia}

To determine whether the bilateral PL or IL cortex plays a role in context-based analgesia in rats, we used an optogenetic technique that enables specific activation of glutamatergic neurons. The behavioral training paradigm is shown in Fig. 5A.

Probe test 1 indicated a clear and stable contextdependent difference in pain perception between contexts in the PL group $(t=3.39, P<0.01, n=10)$. The PLLs of probe test 1 in context $A$ were significantly longer than baseline (matched with LT in training) $(t=2.49, P<$ $0.05)$. These results indicated that an analgesic effect based on cognition of different contexts was successfully established in rats.

Optogenetic activation of pyramidal cells in the PL abolished this context-based analgesic effect $(n=10$, LED-off: $\quad t=4.22, \quad P<0.01$; LED-on: $t=0.12$, $P>0.05$ ) (Fig. 5B), suggesting that the PL cortex is responsible for mediating the context-based analgesic effect. A significant increase of PLLs (context A) in the LED-off group was found compared with baseline $(t=2.30, P<0.05)$ (Fig. 5B).

Similarly, optogenetic activation of pyramidal cells in the IL cortex (LED-on) also abolished this context-based analgesic effect ( $n=11$, LED-off: $t=2.57, P<0.05$; LED-on: $t=0.29, P>0.05$ ) (Fig. 5B).

Together, these data indicate that pyramidal neurons in the prefrontal cortex (in the PL and IL cortices) participate in non-drug dependent, context-based analgesia and that optogenetic activation of these neurons eliminates the established context-based analgesic effect in rats.

\section{Optogenetic Inhibition of the PL or IL Cortex also Eliminated the Context-Based Analgesia}

The behavioral training paradigm is shown in Fig. 6A. Optogenetic inhibition of pyramidal neurons in the PL cortex blocked the context-based analgesic effect $(n=10$, LED-off: $t=2.58, P<0.05$; LED-on: $t=0.12, P>0.05$ ) (Fig. 6B), suggesting that the PL cortex is responsible for mediating this effect. A significant increase of PLLs (context A) in the LED-off group occurred compared with baseline ( $t=2.46, P<0.05$ ) (Fig. 6B).

Similar to the PL cortex, optogenetic inhibition of pyramidal neurons in the IL cortex also blocked the context-based analgesic effect ( $n=11$, LED-off: $t=2.90$, $P<0.01$; LED-on: $t=0.63, P>0.05$ ) (Fig. 6B).

Altogether, these results suggest that pyramidal neurons in the PL and IL cortices participate in the non-drugdependent, context-based analgesia and that optogenetic inhibition of these neurons eliminates the established context-based analgesia effect in rats.

\section{Discussion}

Pain and cognition have close interactions. In the present study, two different contexts (dark and bright) were matched with high $\left(52^{\circ} \mathrm{C}\right)$ or low $\left(48^{\circ} \mathrm{C}\right)$ temperature in the hot-plate test during training. Rats showed longer PLLs in trials with the context originally matched to the LT than those to the HT (Fig. 2), indicating successful 
A

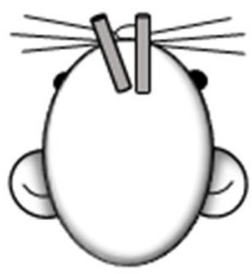

C
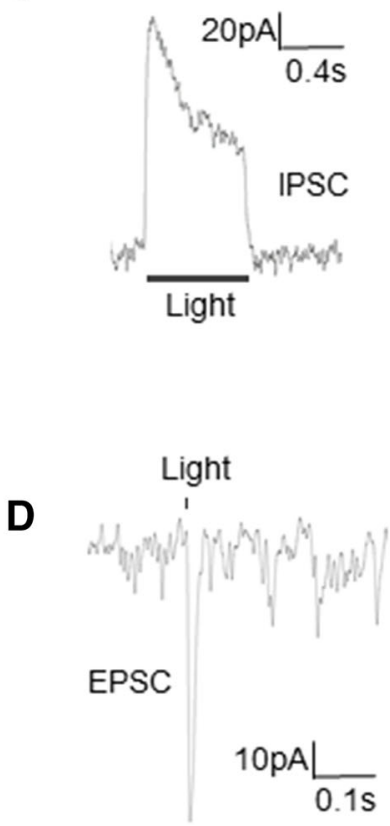

B
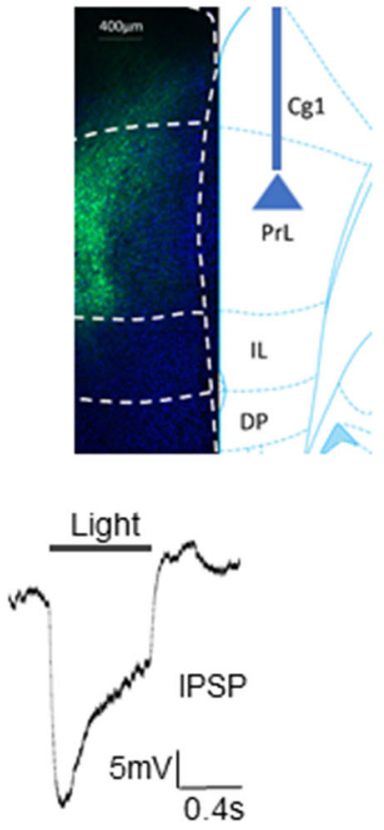

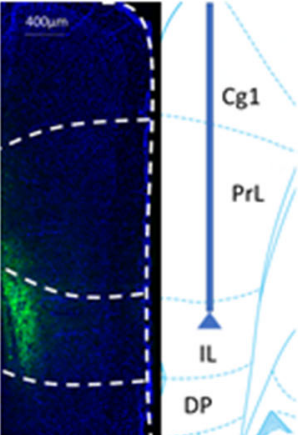

Electrical stimulus

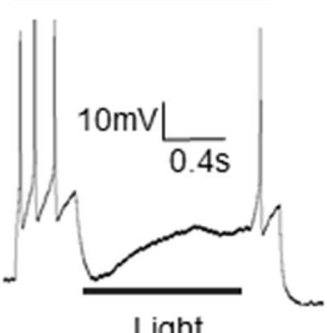

Light
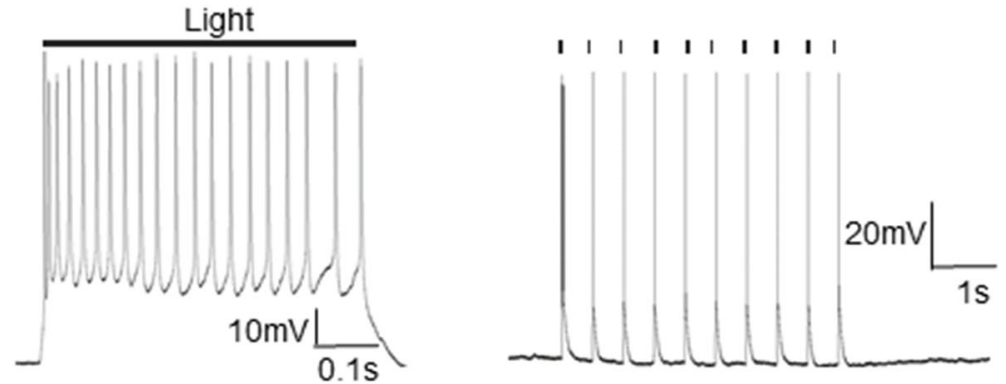

Fig. 4 Confirmation of optogenetic inhibition or inhibition of neuronal firing in pyramidal neurons. A Schematic of the implanted optic fibers: in the left hemisphere tilted $20^{\circ}$, and vertical on the right side. B EYFP expression in excitatory PL/IL neurons after viral injection. C Examples of yellow light-induced outward current and membrane hyperpolarization in a neuron expressing ArchT. An IPSC (left), IPSP (middle), and inhibition of APs were induced by the

establishment of a context-based analgesic effect. This model is a type of placebo-like cognition modulation. Naloxone blockade (Fig. 3) showed that this context-based analgesia is a placebo-like phenomenon.

\section{Animal Model for Cognitive Modulation of Pain}

Pain is a highly subjective perceptual experience that can be affected by cognitive processes [17]. Typical examples include the contextual and emotional modulation of pain as well as placebo effects. Neuroimaging studies have revealed several cortical and subcortical substrates of these effects [18-25]. Support for causal relationships between brain activity and pain modulation has been obtained from yellow light stimulation. D Example of a blue light-evoked EPSC recorded in an EYFP-tagged $\mathrm{ChR} 2$-expressing neuron (left). Current clamp recordings under either continuous blue-light stimulation or in response to blue light delivered at interpulse intervals of $0.5 \mathrm{~s}$. The pulse-locked neuronal firing was induced by the blue light, confirming the expression and function of $\mathrm{ChR} 2$ in the pyramidal neuron (middle and left).

brain stimulation studies, including transcranial direct current stimulation and transcranial magnetic stimulation [26, 27]. However, more in-depth investigation of the cognitive modulation of pain at the neuronal and molecular levels requires back-translation of human studies to animal models, which is not easily established [7].

Several rodent models have been reported, with different training protocols, analgesics, and methods of pain evaluation [7, 8, 28]. Most of them primarily applied a conditioning strategy, by associating the injection of analgesics with specific visual or contextual cues. Later exposure to these cues yielded conditioned placebo or nocebo effects and/or expectation. Another strategy, developed by Ford et al. [29, 30], used novel contexts or objects 
Fig. 5 Optogenetic activation of either PL or IL excitatory neurons blocked the contextbased analgesic effect in rats. A Training and probe paradigm. B Optogenetic activation of neurons in either PL or IL cortex affected PLLs in the hotplate test. Note that the contextbased analgesia was significantly decreased with LED-on but not with LED-off. Context A, black; Context B, grey; HT, high temperature; LT, low temperature. $n=10$ in both PL and IL groups, $* P<0.05$,

$* * P<0.01$, paired $t$ test.

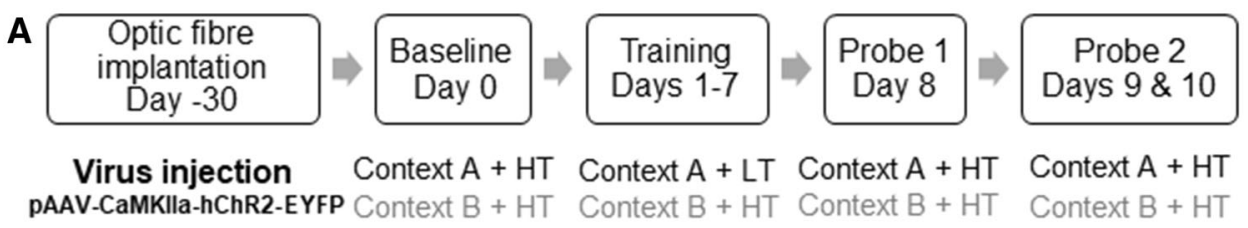

+ Light off/on
B

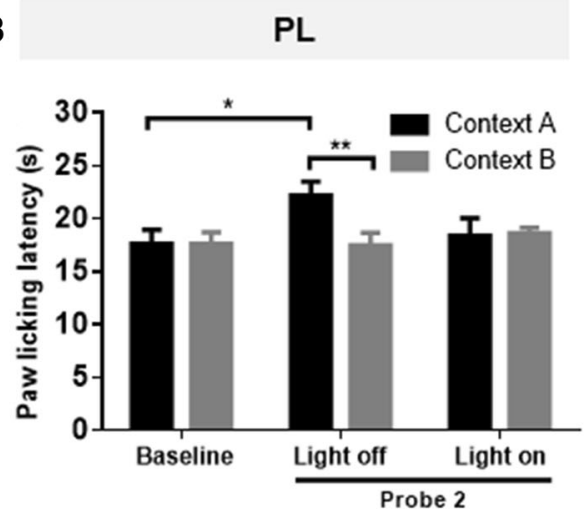

IL

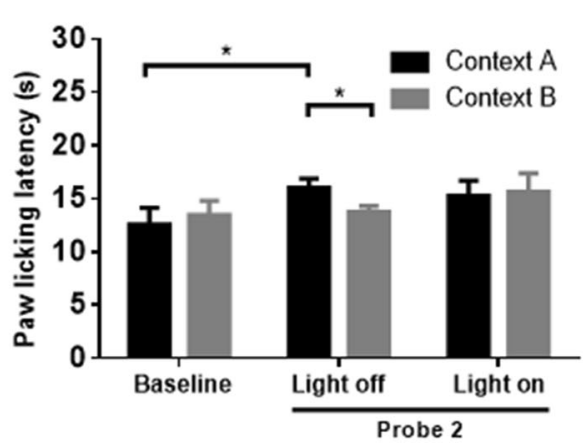

Fig. 6 Optogenetic inhibition of either PL or IL excitatory neurons blocked the contextbased analgesic effect in rats. A Training and probe paradigm. B Optogenetic inhibition of neurons in either the PL or IL cortex abolished the PLL difference between the two contexts in rats showing contextbased analgesia. Note that PLLs were not influenced at light-off during the hot-plate test. Context A, black; Context B, grey; HT, high temperature; LT, low temperature. $n=10$ in the PL group and $n=11$ in the IL group, $* P<0.05$, paired $t$ test.

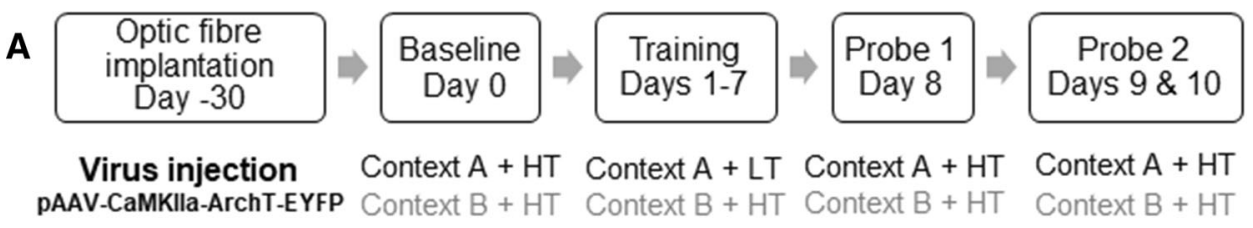

+ Light offlon
B

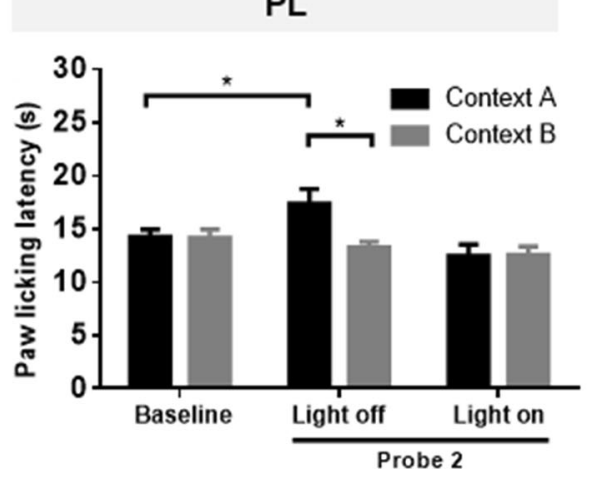

IL

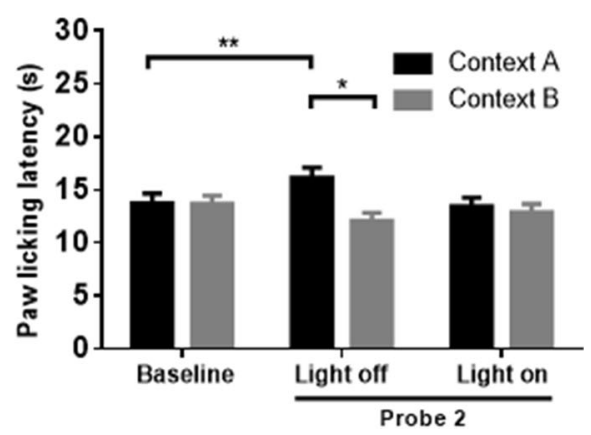

in the testing chamber to distract the animal's attention from pain. This model showed attenuated nociceptive behaviors in the second phase of the formalin test.

In the present study, we developed a novel strategy, by matching two different contextual cues to different testing temperatures in the hot-plate test (Fig. 2). The absence of analgesics in the whole procedure mimics many clinical situations where pure cognitive processes, without any medication, are sufficient to modulate pain. In addition, the hot-plate test is a physiological pain test without persistent injury, enabling multiple probing trials in the same subject. This model also allows flexible revision for experimental necessity. For example, analgesics can be incorporated into the training protocol, and the training phase can be prolonged or repeated to consolidate the strength of modulation. Finally, the self-control strategy for evaluating cognitive modulation bypasses daily fluctuations in the pain threshold without affecting cross-subject differences in the modulatory effect.

\section{Role of the PFC in Context-Based Analgesia}

Pain is a distressing experience associated with actual or potential tissue damage with sensory, emotional, cognitive, 
and social components [31]. The PFC is a crucial integration center for both sensory and emotional pain perception [10, 32-34]. Non-invasive low-frequency repetitive transcranial magnetic stimulation can completely block a context-based analgesic effect [27]. Furthermore, the PFC participates in expectancy-induced changes in subjective pain ratings [35].

In this study, optogenetic light-activation of inhibitory ArchT or excitatory hChR2 in glutaminergic neurons in both the PL and IL cortices blocked the context-related (Figs 5, 6), non-drug-dependent analgesic effect in rats. These results are consistent with a previous report [36]. Interestingly, however, unlike mechanical pain sensitivity, thermal pain sensitivity is changed less by cortical modulation. For example, in a rat model of empathy for pain, which is also dependent on the PL/IL cortices, only mechanical pain hypersensitivity can be demonstrated while thermal pain sensitivity remains unchanged [37, 38]. Intra-PL/IL administration of ethanol has also been demonstrated to result in mechanical pain hypersensitivity but with unchanged thermal pain sensitivity [39].

The differences between the findings of the new model and previous findings may be because the context-based experimental strategy is more dependent on the traininginduced experience rather than emotional impact compared with empathy. The mechanisms of expectation-induced analgesia and empathy-induced pain sensitivity may be different. Several studies have shown that activity due to expectation-induced analgesia in the frontal cortex is associated with pain processing and pain modulation [24]. Since ventromedial PFC (including both IL and PL cortices) activity is associated with reward expectation [40], one possible interpretation of our results is that the cognitive function of the PFC plays a more important role in the expectation-related modulation of pain than in pain sensation. Therefore, it is important to consider the possibility that the integrative function of the $\mathrm{PFC}$ is decisive in context-based analgesia-either activating or silencing the PFC could influence the PFC homeostasis and thus interfere with the cognitive processing of the contextual modulation of pain. So, the cognitive function of the PFC plays a more important role in pain modulation than in pain sensation.

This study is the first to demonstrate context-based analgesia with pinpoint accuracy to the PL and IL cortices. However, the literature suggests that the left and right PFCs may respond to different executive functions [41]. Thus, further research is needed to investigate the role of the PL and IL cortices in the context-based analgesic effect. Understanding the cognitive modulation of pain like that in context-based analgesia is complex, because it involves multiple brain regions that project to the PFC. Our data demonstrate that the role of the $\mathrm{PFC}$ is like an aggregator, thus feasible upstream and downstream structures should also be considered in further studies.

\section{A Novel Context-Based Analgesia Model Mediated by Expectation in Rats}

Both the placebo effect and context-based analgesia are based on previous experience, so we speculate that common features may exist between this new model of context-based analgesia and placebo analgesia. The most well-known theories pertaining to the contextual-modulation analgesic effect are classical conditioning and expectation [42, 43].

Distinct pharmacological mechanisms underlying expectation and conditioning have been revealed by a carefully designed human study, which has shown that expectation triggers endogenous opioids, while conditioning activates specific subsystems not necessarily dependent on the opioid system [44]. Expectation-dependent placebo analgesia mediated by the opioid system has been reliably reported in the literature [45]. Morphine (an opiate analgesic)-induced placebo analgesia is dependent on expectation and can be blocked by opioid antagonists. However, conditioning with nonsteroidal anti-inflammatory drugs elicits opioid-independent analgesia that is only partially mediated by expectation $[6,46]$.

According to the literature, naloxone is an opioid antagonist used in rodent experiments at a dose of $5 \mathrm{mg} /$ $\mathrm{kg}$. Therefore, $5 \mathrm{mg} / \mathrm{kg}$ was selected as an appropriate dose for the current procedure. In our novel model, naloxone significantly blocked the cognitive-mediated analgesia induced by specific contexts and strong or weak thermal stimulation (Fig. 3), implying that the context-based analgesic effect in rats mainly relies on expectation of a strong or weak stimulation based on previous experience rather than conditioning.

Context-based analgesia, or placebo analgesia, is of great interest for future clinical applications. Not only is qualitative research on humans needed, but mechanistic research in animals will also help. This novel rat model will shed new light on our understanding of the brain mechanisms underlying cognition-modulated analgesia as well as non-drug-induced, expectation-dependent placebo analgesia, which will help develop improved treatment strategies for patients in pain.

In conclusion, we have established a non-drug dependent, context-based analgesia model in rats that is endogenous opioid-dependent. The PFC, particularly the PL and the IL cortices, is involved in this analgesic effect. Our study provides a new model for studies of the cognitive modulation of pain as well as placebo-like analgesia in animals. 
Acknowledgments This work was supported by grants from the National Natural Science Foundation of China (91732107, 31200835, 81571067, and 81521063) and the National Basic Research Development Program (973 Program) of China (2014CB548200 and 2015CB554503).

\section{Compliance with Ethical Standards}

Conflict of interest The authors claim no competing interests.

Open Access This article is distributed under the terms of the Creative Commons Attribution 4.0 International License (http:// creativecommons.org/licenses/by/4.0/), which permits unrestricted use, distribution, and reproduction in any medium, provided you give appropriate credit to the original author(s) and the source, provide a link to the Creative Commons license, and indicate if changes were made.

\section{References}

1. Wager TD, Atlas LY. The neuroscience of placebo effects: connecting context, learning and health. Nat Rev Neurosci 2015, 16: 403-418.

2. Moriarty O, Finn DP. Cognition and pain. Curr Opin Support Palliat Care 2014, 8: 130-136.

3. Moriarty O, McGuire BE, Finn DP. The effect of pain on cognitive function: a review of clinical and preclinical research. Prog Neurobiol 2011, 93: 385-404.

4. Attal N, Masselin-Dubois A, Martinez V, Jayr C, Albi A, Fermanian J, et al. Does cognitive functioning predict chronic pain? Results from a prospective surgical cohort. Brain 2014, 137: 904-917.

5. Lee I, Lee B, Park H, Olausson H, Enck P, Chae Y. A new animal model of placebo analgesia: involvement of the dopaminergic system in reward learning. Sci Rep 2015, 5: 17140.

6. Guo J, Wang J, Luo F. Dissection of placebo analgesia in mice: the conditions for activation of opioid and non-opioid systems. J Psychopharmacol 2010, 24: 1561-1567.

7. McNabb CT, White MM, Harris AL, Fuchs PN. The elusive rat model of conditioned placebo analgesia. Pain 2014, 155: 2022-2032.

8. Nolan TA, Price DD, Caudle RM, Murphy NP, Neubert JK. Placebo-induced analgesia in an operant pain model in rats. Pain 2012, 153: 2009-2016.

9. Benn A, Barker GR, Stuart SA, Roloff EV, Teschemacher AG. Optogenetic stimulation of prefrontal glutamatergic neurons enhances recognition memory. J Neurosci 2016, 36: 4930-4939.

10. Zhang Z, Gadotti VM, Chen L, Souza IA, Stemkowski PL, Zamponi GW. Role of prelimbic GABAergic circuits in sensory and emotional aspects of neuropathic pain. Cell Rep 2015, 12: 752-759.

11. Kilkenny C, Browne WJ, Cuthill IC, Emerson M, Altman DG. Improving bioscience research reporting: the ARRIVE guidelines for reporting animal research. PLoS Biol 2010, 8: e1000412.

12. Yue L, Ma LY, Cui S, Liu FY, Yi M, Wan Y. Brain-derived neurotrophic factor in the infralimbic cortex alleviates inflammatory pain. Neurosci Lett 2017, 655: 7-13.

13. Wang GQ, Cen $\mathrm{C}$, Li C, Cao S, Wang N, Zhou Z, et al. Deactivation of excitatory neurons in the prelimbic cortex via Cdk5 promotes pain sensation and anxiety. Nat Commun 2015, 6: 7660.

14. Zhang C, Chen RX, Zhang Y, Wang J, Liu FY. Reduced GABAergic transmission in the ventrobasal thalamus contributes to thermal hyperalgesia in chronic inflammatory pain. Sci Rep 2017, 7: 41439.

15. Eippert F, Bingel U, Schoell ED, Yacubian J, Klinger R, Lorenz $\mathrm{J}$, et al. Activation of the opioidergic descending pain control system underlies placebo analgesia. Neuron 2009, 63: 533-543.

16. Bernstein JG, Garrity PA, Boyden ES. Optogenetics and thermogenetics: technologies for controlling the activity of targeted cells within intact neural circuits. Curr Opin Neurobiol 2012, 22 : 61-71.

17. Moriarty O, Finn DP. Cognition and pain. Curr Opin Support Pa 2014, 8: 130-136.

18. Faria V, Fredrikson M, Furmark T. Imaging the placebo response: A neurofunctional review. Eur Neuropsychopharm 2008, 18: 473-485.

19. Lidstone SCC, Stoessl AJ. Understanding the placebo effect: contributions from neuroimaging. Mol Imaging Biol 2007, 9: 176-185.

20. Ploghaus A, Narain C, Beckmann CF, Clare S, Bantick S, Wise $\mathrm{R}$, et al. Exacerbation of pain by anxiety is associated with activity in a hippocampal network. J Neurosci 2001, 21: 9896-9903.

21. Ploner M, Lee MC, Wiech K, Bingel U, Tracey I. Flexible cerebral connectivity patterns subserve contextual modulations of pain. Cereb Cortex 2011, 21: 719-726.

22. Quevedo AS, Coghill RC. Attentional modulation of spatial integration of pain: evidence for dynamic spatial tuning. J Neurosci 2007, 27: 11635-11640.

23. Wager TD. Expectations and anxiety as mediators of placebo effects in pain. Pain 2005, 115: 225-226.

24. Wager TD, Rilling JK, Smith EE, Sokolik A, Casey KL, Davidson RJ, et al. Placebo-induced changes in FMRI in the anticipation and experience of pain. Science 2004, 303: 1162-1167.

25. Yi M, Zhang H. Nociceptive memory in the brain: cortical mechanisms of chronic pain. J Neurosci 2011, 31: 13343-13345.

26. Egorova N, Yu R, Kaur N, Vangel M, Gollub RL, Dougherty DD, et al. Neuromodulation of conditioned placebo/nocebo in heat pain: anodal vs cathodal transcranial direct current stimulation to the right dorsolateral prefrontal cortex. Pain 2015, 156: $1342-1347$.

27. Krummenacher P, Candia V, Folkers G, Schedlowski M, Schonbachler G. Prefrontal cortex modulates placebo analgesia. Pain 2010, 148: 368-374.

28. Bryant CD, Roberts KW, Culbertson CS, Le A, Evans CJ, Fanselow MS. Pavlovian conditioning of multiple opioid-like responses in mice. Drug Alcohol Depend 2009, 103: 74-83.

29. Ford GK, Moriarty O, McGuire BE, Finn DP. Investigating the effects of distracting stimuli on nociceptive behaviour and associated alterations in brain monoamines in rats. Eur $\mathbf{J}$ Pain 2008, 12: 970-979.

30. Ford GK, Moriarty O, Okine BN, Tully E, Mulcahy A, Harhen B, et al. Involvement of the endocannabinoid system in attentional modulation of nociceptive behaviour in rats. Eur J Pain 2015, 19: $1177-1185$

31. Williams AC, Craig KD. Updating the definition of pain. Pain 2016, 157: 2420-2423.

32. Kong J, Jensen K, Loiotile R, Cheetham A, Wey HY, Tan Y, et al. Functional connectivity of the frontoparietal network predicts cognitive modulation of pain. Pain 2013, 154: 459-467.

33. Schulz E, May ES, Postorino M, Tiemann L, Nickel MM, Witkovsky $\mathrm{V}$, et al. Prefrontal gamma oscillations encode tonic pain in humans. Cereb Cortex 2015, 25: 4407-4414.

34. Apkarian AV, Bushnell MC, Treede RD, Zubieta JK. Human brain mechanisms of pain perception and regulation in health and disease. Eur J Pain 2005, 9: 463-484. 
35. Haanstra TM, Kamper SJ, Williams CM, Spriensma AS, Lin CW, Maher CG, et al. Does adherence to treatment mediate the relationship between patients' treatment outcome expectancies and the outcomes of pain intensity and recovery from acute low back pain? Pain 2015, 156: 1530-1536.

36. Wiech K. Deconstructing the sensation of pain: The influence of cognitive processes on pain perception. Science 2016, 354: 584-587.

37. Li Z, Lu YF, Li CL, Wang Y, Sun W, He T, et al. Social interaction with a cagemate in pain facilitates subsequent spinal nociception via activation of the medial prefrontal cortex in rats. Pain 2014, 155: 1253-1261.

38. Lu YF, Yang Y, Li CL, Wang Y, Li Z, Chen J. The locus coeruleus-norepinephrine system mediates empathy for pain through selective up-regulation of $\mathrm{P} 2 \mathrm{X} 3$ receptor in dorsal root ganglia in rats. Front Neural Circuits 2017, 11: 66.

39. Geng KW, He T, Wang RR, Li CL, Luo WJ, Wu FF, et al. Ethanol increases mechanical pain sensitivity in rats via activation of GABAA receptors in medial prefrontal cortex. Neurosci Bull 2016, 32: 433-444.

40. Briones RM, Miranda JM, Mellado-Gil JM, Castro MJ, Gonzalez-Molina M, Cuesta-Munoz AL, et al. Differential analysis of donor characteristics for pancreas and islet transplantation. Transplant Proc 2006, 38: 2579-2581.

41. Cipolotti L, Spano B, Healy C, Tudor-Sfetea C, Chan E, White $\mathrm{M}$, et al. Inhibition processes are dissociable and lateralized in human prefrontal cortex. Neuropsychologia 2016, 93: 1-12.

42. Peerdeman KJ, van Laarhoven AI, Peters ML, Evers AW. An integrative review of the influence of expectancies on pain. Front Psychol 2016, 7: 1270.

43. Reicherts P, Gerdes ABM, Pauli P, Wieser MJ. Psychological placebo and nocebo effects on pain rely on expectation and previous experience. J Pain 2016, 17: 203-214.

44. Amanzio M, Benedetti F. Neuropharmacological dissection of placebo analgesia: expectation-activated opioid systems versus conditioning-activated specific subsystems. J Neurosci 1999, 19: 484-494.

45. Schafer SM, Geuter S, Wager TD. Mechanisms of placebo analgesia: A dual-process model informed by insights from crossspecies comparisons. Prog Neurobiol 2018, 160: 101-122.

46. Benedetti F, Pollo A, Lopiano L, Lanotte M, Vighetti S, Rainero I. Conscious expectation and unconscious conditioning in analgesic, motor, and hormonal placebo/nocebo responses. J Neurosci 2003, 23: 4315-4323. 